\title{
Neutral Hydrogen in Galaxies from Low to High Redshift
}

\author{
S-L. Blyth*, A. Bouchard, K.J. van der Heyden, W.J.G. de Blok, R.C. \\ Kraan-Korteweg, M. Ramatsoku \\ Department of Astronomy, University of Cape Town, Private Bag X3, Rondebosch, Cape Town, \\ 7701, South Africa \\ E-mail: sarblyth@ast.uct.ac.za

\section{W. van Driel} \\ GEPI, Observatoire de Paris, CNRS, Université Paris Diderot, 5 place Jules Janssen, 92190 \\ Meudon, France
}

\begin{abstract}
HI spectral stacking techniques have recently been used to isolate the average signal from coadded low signal-to-noise observations of intermediate redshift galaxies. We exploit these methods to extract $\mathrm{HI}$ information for galaxies which were classified as non-detections (i.e. $\leq 3 \sigma)$ in the Nançay Interstellar Baryon Legacy Extragalactic Survey (NIBLES). We present the average HI content of the non-detected galaxies and investigate them further by selecting subsamples according to a colour cut corresponding to different morphologies with the aim to learn more about the Hi distribution in galaxies in the Local Volume.

The use of these techniques is also extended to outline possible survey scenarios for a deep HI survey using MeerKAT. Future radio surveys aiming to quantify the average Hi content of the universe over cosmic time will rely on HI stacking and co-adding techniques in order to push to the lowest mass limits achievable within reasonable observing timescales. We present preliminary stacking results based on simulated data for three survey scenarios using MeerKAT.
\end{abstract}

Panoramic Radio Astronomy: Wide-field 1-2 GHz research on galaxy evolution - PRA2009

June 02 - 052009

Groningen, the Netherlands

\footnotetext{
* Speaker.
} 


\section{Introduction}

One of the key unanswered questions currently facing HI astrophysics is how galaxies evolve over cosmic time. In order to work towards answering this question, we need to investigate how the cosmic density of neutral gas, $\Omega_{\text {gas }}$, the fuel for star formation, evolves over cosmic time. It is also imperative to study how neutral hydrogen is distributed among galaxies, also known as the HI mass function (HIMF), and how this distribution changes as a function of lookback time in the universe.

The cosmic neutral gas density has been measured for $0.6<z<5$ using damped Lyman-alpha observations [1,2] and using direct HI observations of galaxies in the local universe at $z=0$ [3]. Most recently, further measurements of $\Omega_{\text {gas }}$ using Hi observations of galaxies have been made at intermediate redshifts, $0<z<1$ [4]. However, due to the difficulty in performing these measurements and the limitations of current radio observing facilities, the error bars on the intermediate redshift results are sufficiently large that we are not yet able to clearly discern the evolution of $\Omega_{\text {gas }}$ from low to high redshifts. Further, the HIMF is currently known only for the local universe and it is therefore of great interest to measure this distribution at higher redshifts in the near future.

Due to the intrinsic weakness of the Hi line, in order to measure this emission at cosmological distances we need different techniques to enable optimisation of observing times, as well as larger more sensitive radio telescopes with large fields of view and wide band frequency coverage. Currently, one of the highest redshift galaxies yet measured in $21 \mathrm{~cm}$ emission is at $z=0.21$ and required an observing time of $\sim 20 \times 12$ hours on the Westerbork Synthesis Radio Telescope (WSRT) [5]. A technique being employed by several groups [6, 7, 5, 4] to measure Hi at intermediate redshifts and optimise observing times is the method of HI spectral stacking or co-adding. Using known redshifts measured by some independent means, for the observed galaxies, their radio spectra are shifted such that the HI lines fall into a common channel/s and then the weighted mean of the spectra can be calculated. In this way, many low signal-to-noise observations can be used to find a higher signal-to-noise average signal and hence an average mass or density of HI at a particular redshift using more reasonable observing times. The issue of larger, more sensitive telescopes is being addressed by the construction of the Square Kilometre Array (SKA) and its precursor instruments, MeerKAT [9] and the Australian SKA Pathfinder, ASKAP [10].

In these proceedings we introduce the CRUMBS (Characterizing Radio-Undetected Masses in Baryonic Surveys) project which investigates the use of HI stacking techniques as applied to nearby galaxies classified as non-detections in the Nançay Interstellar Baryon Legacy Extragalactic Survey (NIBLES) [8]. Our aim is to quantify the $\left\langle M_{H I}\right\rangle$ contained in these galaxies and investigate possible dependencies on optical morphology and other parameters. We then extend the use of these techniques to outline possible survey scenarios for a deep HI survey using MeerKAT.

\section{Hi at low $z$ : NIBLES and CRUMBS}

One of the primary aims of the NIBLES survey is to find and quantify the density of baryons in the Local Universe. NIBLES is a targeted Hi survey of 3000 galaxies in the Local Universe $(900<c z<12000 \mathrm{~km} / \mathrm{s})$ from the Sloan Digital Sky Survey (SDSS) covering a range of morphological types [11]. The goal of the CRUMBS project is to quantify or set a limit on the $\left\langle M_{H I}\right\rangle$ of 

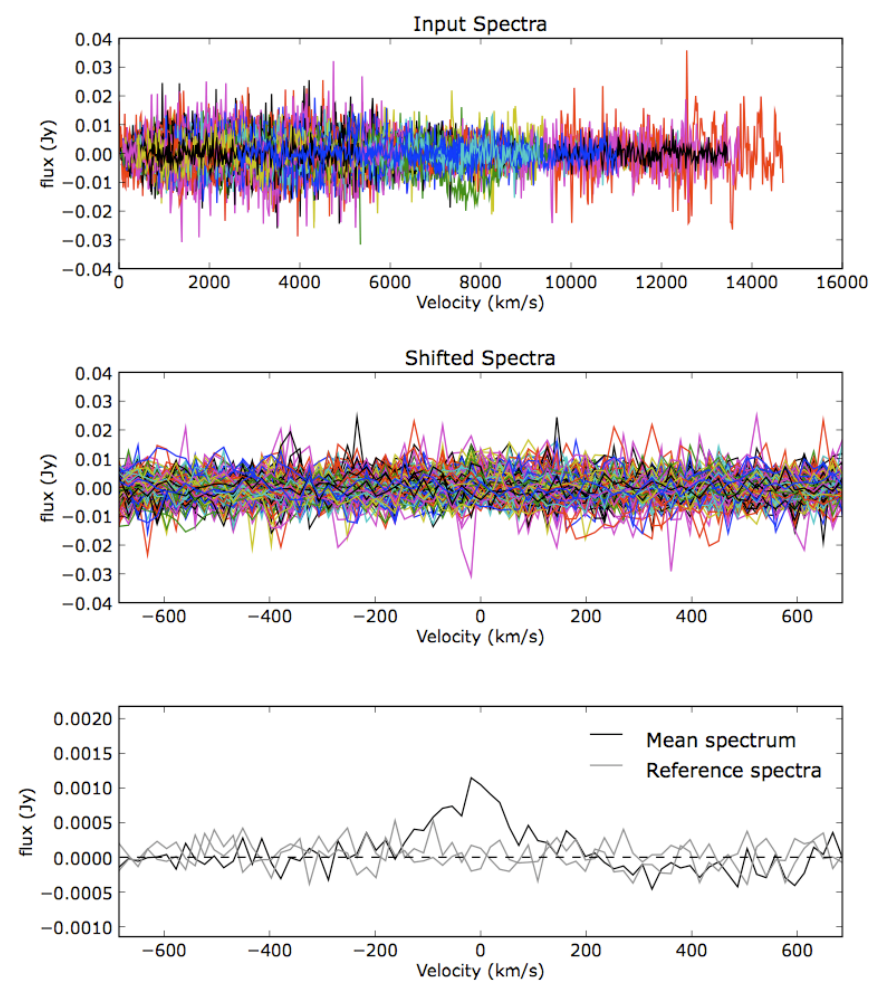

Figure 1: Upper panel: Input HI spectra (flux vs. velocity) for non-detected NIBLES sources. Middle panel: Spectra from top panel after shifting according to known redshifts. Lower panel: Final average spectrum (black line) computed by co-adding shifted spectra from middle panel. The reference spectra are shown in grey.

galaxies which are classified as non-detections in NIBLES. Here we define a non-detection as an $\mathrm{HI}$ spectrum where the peak signal to noise value is $<3$.

In these proceedings we present preliminary results based on 884 NIBLES galaxies. Of these, a total of 337 were classified as non-detections. We used the optical redshifts from the SDSS to shift and co-add the non-detections and recovered a significant averaged signal corresponding to a $\left\langle M_{H I}\right\rangle=1.03 \times 10^{8} M_{\odot}$ (for $\left\langle D_{L}\right\rangle=52 \pm 2 \mathrm{Mpc}$ ). Figure 1 shows the 337 input spectra (upper panel), the spectra after they are shifted such that their HI lines should fall into the $0 \mathrm{~km} / \mathrm{s}$ velocity channel (middle panel) and the final average spectrum (black solid line, lower panel). Also shown in the bottom panel of the figure are two 'reference' spectra (grey lines) which are computed using the same input spectra, but in this case each spectrum is shifted according to a randomly assigned redshift value before being co-added.

In order to investigate further the characteristics of our sample of non-detections, we subdivided them by various criteria: morphology/colour (red vs. blue according to the definition from [12]), $g-i$ colour, absolute $g$-band magnitude and radius. The red vs. blue colour cut 

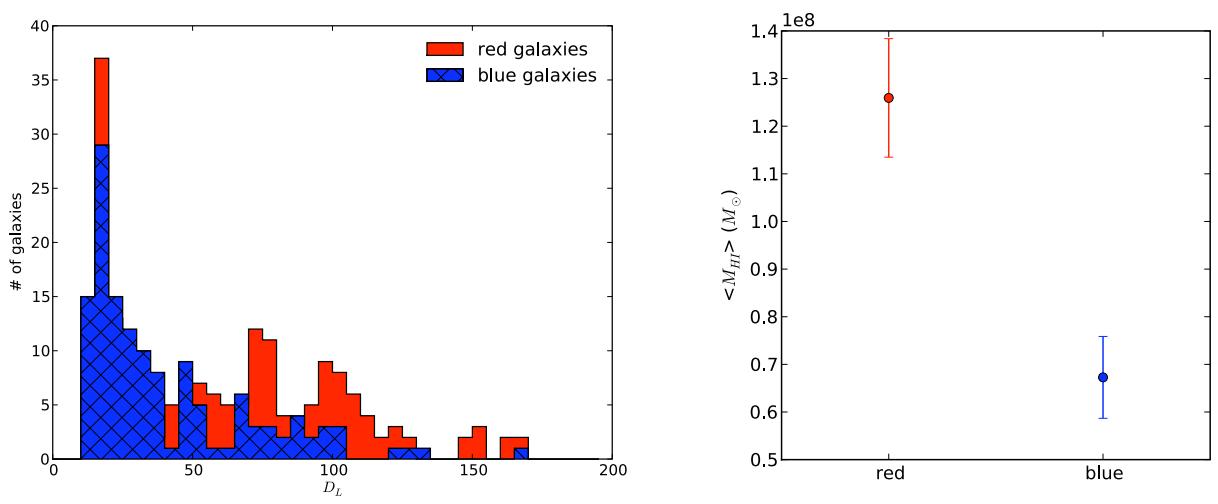

Figure 2: Left: Luminosity distance distribution for the red (solid histogram) and blue (hatched histogram) non-detected galaxy samples. Right: $\left\langle M_{H I}\right\rangle$ computed after co-adding for the red and blue subsamples.

from [12] has been shown to separate SDSS galaxies into early- vs. late-type subsamples respectively, enabling a rough morphology cut. The left panel of Figure 2 shows the luminosity distance histograms, $D_{L}$, (calculated using SDSS redshifts) for the red/early-type galaxies (solid histogram) and blue/late-type galaxies (hatched histogram). There is a difference in the distributions with most of the blue galaxies being observed at smaller $D_{L}$ while the red galaxies have a secondary peak between $50<D_{L}<100 \mathrm{Mpc}$. These different distributions explain why the resulting coadded $\left\langle M_{H I}\right\rangle$ for the red galaxies is greater than for the blue galaxies (shown in the right panel of Figure 2). One would expect that the blue galaxies should contain more HI, however, our sample consists of non-detections; therefore, the blue galaxies in the sample are already unusual and relatively gas-poor, and the red galaxies which are contributing to the signal are likely large ellipticals since they are mostly located at larger distances.

Further cuts on magnitude and size vs. $\left\langle M_{H I}\right\rangle$ are currently being investigated and will be presented elsewhere [13].

\section{HI at higher $z$ with MeerKAT}

We have extended the use of the HI co-adding technique to estimate optimal observing scenarios to measure $\Omega_{\text {gas }}$ using a MeerKAT deep HI survey. We used the simulated galaxy catalogue from [14] that simulates the cosmic evolution of neutral gas based on the virtual galaxy catalogue by De Lucia et al. $[15,16]$. We used the catalogue galaxies' Hi properties to simulate line profiles for galaxies with redshifts $0<z<2$ and within a field of view of $0.47 \times 0.47$ degrees $(25 \%$ of the expected MeerKAT field of view). We assumed perfect optical data or, in other words, that the redshifts for all the galaxies in our simulated sample were known and accurate. This was to ensure we could verify that the co-adding method worked, before introducing additional complications such as inclination effects, magnitude biases or redshift errors.

Three survey scenarios for single pointing observations were simulated using sensitivities based on the MeerKAT 80 antenna configuration presented in [17]. The expected noise RMS values for each survey scenario are presented in Table 1. 


\begin{tabular}{cc}
\hline Survey Scenario & Expected Noise RMS $(\mathbf{m J y})$ \\
\hline $\begin{array}{c}3 \text { months } \\
(90 \text { days } \times 12 \text { hours })\end{array}$ & $2.55 \times 10^{-2}$ \\
\hline $\begin{array}{c}6 \text { months } \\
(180 \text { days } \times 12 \text { hours })\end{array}$ & $1.81 \times 10^{-2}$ \\
\hline $\begin{array}{c}1 \text { year } \\
(365 \text { days } \times 12 \text { hours })\end{array}$ & $1.27 \times 10^{-2}$ \\
\hline
\end{tabular}

Table 1: Potential survey scenarios for a deep MeerKAT HI survey and the corresponding noise RMS values.

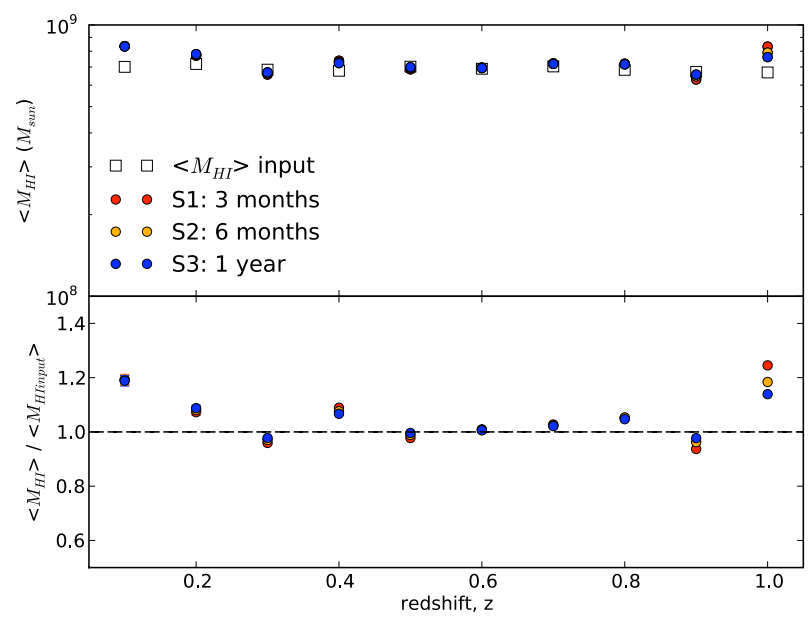

Figure 3: Top panel: $\left\langle M_{H I}\right\rangle$ vs. redshift for the simulated input galaxies (white squares), and recovered $\left\langle M_{H I}\right\rangle$ for the 3 survey scenarios (3 months: red circles, 6 months: orange circles, 1 year: blue circles). Bottom panel: Ratio of recovered $\left\langle M_{H I}\right\rangle$ to input $\left\langle M_{H I}\right\rangle$ values as a function of redshift for each survey scenario. Symbols are the same as in the top panel.

The recovered $\left\langle M_{H I}\right\rangle$ values and comparison to the input values for the three survey scenarios are presented in Figure 3. The stacking method recovers the input $\left\langle M_{H I}\right\rangle$ values well over a wide range of redshifts, and does better for longer observing times. Further work is yet to be done on making our simulations more realistic by including effects of different galaxy inclinations and by introducing biases caused by magnitude limited redshift surveys.

\section{Summary}

HI stacking techniques are a promising tool to use for HI surveys, where optical redshifts are already known, to wring out additional information from non-detected sources. Further work is being performed on the NIBLES dataset to extract information on the non-detected galaxies and investigate relationships between $\left\langle M_{H I}\right\rangle$ and quantities such as magnitude, colour, morphology and size. 
Based on preliminary results using simulations we can infer that these techniques will also be successful when applied to deep Hi observations using MeerKAT with the goal to measure the cosmic neutral gas density as a function of redshift. The optimal observing scenario/s are yet to be defined and will be based on simulations eventually including known limitations of spectroscopic redshift catalogues.

\section{References}

[1] Prochaska J. X., Herbert-Fort S. \& Wolfe A. M., 2005, ApJ, 635, 123

[2] Rao S. M., Turnshek D. A., \& Nestor D. B., 2006, ApJ, 636, 610

[3] Zwaan, M. A., Meyer, M. J., Staveley-Smith, L., \& Webster, R. L. 2005, MNRAS, 359, L30

[4] Lah P. et al., 2005, MNRAS, 376, 1357-1366

[5] Verheijen M. et al., 2007, ApJ, 668, L9-L13

[6] Zwaan, M. A. 2000, Ph.D. Thesis

[7] Chengalur, J. N., Braun, R., \& Wieringa, M. 2001, A\&A, 372, 768

[8] van Driel W., Schneider S., Lehnert M., the NIBLES Consortium, in proceedings of "Galaxies in the Local Volume", Sydney, 8-13 July 2007

[9] www.ska.ac.za/meerkat

[10] www.atnf.csiro.au/projects/askap

[11] van Driel W., in proceedings, PRA2009

[12] Baldry I. K., et al., 2004, ApJ, 600, 681

[13] Bouchard A., Blyth S-L. et al., in preparation

[14] Obreschkow D., et al., 2009, ApJ, 698, 1467-1484

[15] de Lucia G., Blaizot J., 2007, MNRAS, 375, 2

[16] Springel V., et al., 2005, Nature, 435, 629

[17] de Blok, W.J.G in proceedings PRA2009 